\title{
Risikofaktor Schimmelpilze: Die meisten Fragen sind offen
}

Sensationelle Pressemeldungen haben die Menschen verunsichert. Erobern tatsächlich gefährliche Pilze Wohnungen und Biotonnen? Lauern tückische Aspergillen in der Blumenerde und gefährden die Lungen argloser Pflanzenfreunde? Sicher ist vor allem eins: Die Erregung der Gemüter steht in krassem Gegensatz. zum eklatanten Mangel an gesicherten Erkenntnissen. H.P. Seidl, Klinik und Poliklinik für Dermatologie und Allergologie am Biederstein der TU München, nahm das Problem unter die Lupe.

ns Kreuzfeuer der Pilzbeschwörer geraten ist vor allem die biologische Komposterzeugung. Das Bundesgesundheitsamt nahm sich der Problematik schon 1991 an. Es warnte allergiegefährdete und abwehrgeschwächte Personen vor Kontakt mit der Biotonne, forderte kurze Entleerungsintervalle und Warnhinweise auf Biokompost. Protest kam sowohl von seiten der Wissenschaft als auch aus der Entsorgungswirtschaft. Schnellschüsse statt wissenschaftlicher Erkenntnis? Die kontroverse Diskussion hält weiter an.

\section{Schimmelpilze sind allgegenwärtig}

Fest steht, daß die gefürchteten „Schimmelpilze“, eine große, heterogene Familie von Sporenbildnern, ubiquitär vorkommen und somit praktisch zur natürlichen Umwelt des Menschen gehören. Bestimmte Arten besitzen infektiöses und allergenes Potential. Rund 200 Arten produzieren Mykotoxine, die oral, über die Atemwege und auch durch Hautkontakt aufgenommen werden können.

Schwieriger zu beantworten ist die Frage, was genau unter erhöhter „Pilzbelastung “ zu verstehen ist. Dazu gibt es, so Seidl, je nach Meßmethode ganz unterschiedliche Angaben. Meist gelten Werte zwischen $10^{2}$ und $10^{4} / \mathrm{m}^{3}$ als normal, manchmal aber auch noch solche von $10^{7} / \mathrm{m}^{3}$. In der Raumluft ist die Konzentration in aller Regel nur halb so hoch wie in der Außenluft. Ist sie höher, muß nach einer Quelle gefahndet werden.

\section{Mehr Meinungen als Beweise}

Vollends auf unsicherem Boden bewegt man sich, wenn es um den $\mathrm{Zu}$ sammenhang zwischen gesundheitlichen Beschwerden und der Schimmelpilzsporenkonzentration geht. Hier

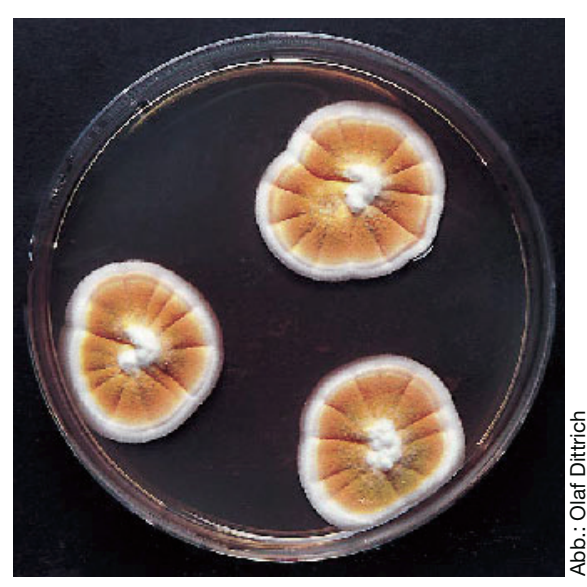

Aspergillus spec. auf Kimmig-Agar. gibt es mehr Meinungen und Empfindungen als tatsächlich Bewiesenes. Publiziert wurden, so Seidl, die unterschiedlichsten Werte. Grenzwerte lassen sich zur Zeit nicht festlegen, weil die Meßmethoden nicht standardisiert sind. Außerdem ist man sich über die Leitkeime uneins. Was völlig fehlt, sind klinische Studien.

\section{Gefahr nur für Prädisponierte}

Prinzipiell möglich sind Pilzinfektionen der Atemwege, seltener der Haut und Nägel, IgE-vermittelte allergische Erkrankungen durch Schimmelpilze sowie Erkrankungen durch Mykotoxine. Systemmykosen nach Infektion über die Atemwege sind laut Seidl ,in der Regel“ auf prädisponierte Personen beschränkt. Für Verunsicherung sorgen allerdings immer mal wieder Fälle, in denen Patienten aus völliger Gesundheit heraus an einer systemischen Pilzinfektion erkranken, wie im Fall eines 16jährigen mit multiplen Aspergillus-Abszessen, der in einem Tierpark arbeitete. Die in der Literatur gern zitierte exogen-allergische Alveolitis, für die Schimmelpilzsporen ohnehin lediglich als eine der möglichen Ursachen in Frage kommen, ist eher eine Rarität.

Das wenig befriedigende Fazit: ,Zum Zusammenhang zwischen Pilzbelastung und Erkrankungen sind die meisten Fragen offen“, so Seidl. Sinnvoll sei es aber, Vorsicht walten zu lassen und die Belastung zu minimieren. Besonders gefährdete prädisponierte Menschen sollten belastete Bereiche meiden. Die Entleerung von Biotonnen und die Arbeit im Kompostwerk gehören sicherlich dazu. Ob eine Gefahr besteht und wie groß die ist, bleibt zu klären.

(ue)

Nach einem Vortrag beim Kongreß „Fortschritte der Allergologie, Immunologie und Dermatologie“, Davos, 10. bis 14.
September 1997. 